\title{
Response to: A double-edged sword: does highly active antiretroviral therapy contribute to syphilis incidence by impairing immunity to Treponema pallidum
}

\author{
David V. Glidden, Ph.D., \\ University of California, San Francisco, San Francisco, CA, USA \\ Kenneth H. Mayer, M.D., and \\ Fenway Institute and Harvard Medical School, Boston, MA, USA \\ Robert M. Grant, M.D., M.P.H. \\ Gladstone Institute of Virology and San Francisco AIDS Foundation, San Francisco, California, \\ USA
}

\begin{abstract}
Randomized double-blind trials of pre-exposure prophylaxis (PrEP) for HIV prevention [1] provide a rigorous test of the author's hypothesis. The methodological strength includes an unconfounded and clearly unexposed control group and an exposed group which received an agent that would putatively increase susceptibility - tenofovir disoproxil fumarate (TDF) co-formulated with emtricitabine (FTC). A unique strength is that these trials were blinded and PrEP was unproven that the time trials were undertaken. Hence, we would not expect that the exposed group would adopt higher risk practices.
\end{abstract}

An analysis of the iPrEx trial [2], a randomized PrEP trial in men who have sex with men/ trans women, found [1] a relative rate of syphilis acquisition for TDF/FTC of 1.14 with a 0.95 confidence interval ( 0.90 to 1.45 ) compared to placebo. Incident syphilis, can be difficult to detect and the relative incidence among those with a negative rapid plasma reagin titer at screening was $1.03,0.95 \mathrm{CI}(0.76$ to 1.38$)$. Adherence, was low in the iPrEx study and when pharmacology is taken into account, the hazard ratio for FTC/TDF among those with drug detected in plasma was 1.00, $0.95 \mathrm{CI}$ (0.62 to 1.61), compared to placebo. Finally, we found that the incidence of syphilis decreased during the period of the trial in the FTC/TDF group (from 6.3 per 100 person years in the first year to 3.7 per 100 person years in subsequent years).

Taken together, it is unlikely TDF/FTC effect on syphilis acquisition would be missed when effects on HIV replication, bone mineral density, symptoms, and kidney function were readily detected in the iPrEx study even in the presence of low adherence. Randomized controlled trials are the gold standard in clinical research and this PrEP trial provides formidable evidence against the authors hypothesis.

Corresponding Author: David V. Glidden, Ph.D., Department of Epidemiology and Biostatistics; University of California, San Francisco, 550 16th St., San Francisco, CA 94158 Phone: +1 (415) 476-1111, Ext. 13603, Fax: +1 (415) 514-8150, david.glidden@ucsf.edu. 


\section{References}

1. Solomon MM, Mayer KH, Glidden DV. Syphilis predicts HIV incidence among men and transgender women who have sex with men in a preexposure prophylaxis trial. Clin Infect Dis. 2014; 59:1020-6. DOI: 10.1093/cid/ciu450 [PubMed: 24928295]

2. Grant RM, Lama JR, Anderson PL, et al. Preexposure chemoprophylaxis for HIV prevention in men who have sex with men. N Engl J Med. 2010; 363:2587-99. DOI: 10.10/56NEJMoa1011205 [PubMed: 21091279] 